\title{
Post-ischemic inflammation regulates neural damage and protection
}

\author{
Takashi Shichita ${ }^{1,2}$, Minako Ito ${ }^{1}$ and Akihiko Yoshimura ${ }^{\text {* }}$ \\ Department of Microbiology and Immunology, School of Medicine, Keio University, Tokyo, Japan \\ 2 Precursory Research for Embryonic Science and Technology, Japan Science and Technology Agency, Tokyo, Japan
}

\section{Edited by:}

Arthur Liesz, University Hospital

Munich, Germany

\section{Reviewed by:}

Mathias Gelderblom, University Medical Center Hamburg-Eppendorf, Germany

Alexander Dressel, University Medicine Greifswald, Germany

\section{*Correspondence:}

Akihiko Yoshimura, Department of Microbiology and Immunology, School of Medicine, Keio University, 35 Shinanomachi, Shinjuku-ku, Tokyo 160-8582, Japan

e-mail:yoshimura@a6.keio.jp

\begin{abstract}
Post-ischemic inflammation is important in ischemic stroke pathology. However, details of the inflammation process, its resolution after stroke and its effect on pathology and neural damage have not been clarified. Brain swelling, which is often fatal in ischemic stroke patients, occurs at an early stage of stroke due to endothelial cell injury and severe inflammation by infiltrated mononuclear cells including macrophages, neutrophils, and lymphocytes. At early stage of inflammation, macrophages are activated by molecules released from necrotic cells [danger-associated molecular patterns (DAMPs)], and inflammatory cytokines and mediators that increase ischemic brain damage by disruption of the blood-brain barrier are released. After post-ischemic inflammation, macrophages function as scavengers of necrotic cell and brain tissue debris. Such macrophages are also involved in tissue repair and neural cell regeneration by producing tropic factors. The mechanisms of inflammation resolution and conversion of inflammation to neuroprotection are largely unknown. In this review, we summarize information accumulated recently about DAMPinduced inflammation and the neuroprotective effects of inflammatory cells, and discuss next generation strategies to treat ischemic stroke.
\end{abstract}

Keywords: damage-associated molecular patterns (DAMPs), inflammation, cytokines, inflammasome, resolution of inflammation

\section{INTRODUCTION}

Inflammation is implicated in almost all of central nervous system (CNS) diseases (Lo, 2010; Moskowitz et al., 2010; Iadecola and Anrather, 2011). Neurodegeneration, infection, trauma, and ischemia stimulate immune responses in the brain, although to varying degrees. The process in neuronal injury involves various intracellular mechanisms (abnormal metabolism and degeneration of protein, dysfunction of organelles, etc.), which cause the activation of microglia and the infiltration of circulating immune cells (Lo, 2010). Inflammation may not be always main process in the pathology of CNS diseases; nonetheless, the distinct characteristics of ischemic stroke are large amount of necrotic neuronal death and extreme infiltration of immune cells (Moskowitz et al., 2010; Iadecola and Anrather, 2011).

Severe inflammation causes cerebral swelling, which is often fatal in ischemic stroke patients. Broad necrotic lesion generates abundant inflammatory mediators and damage-associated molecular patterns (DAMPs), which enhance the chemotaxis of circulating immune cells and make them more efficient participants to promote inflammation (Moskowitz et al., 2010; Iadecola and Anrather, 2011). Cerebral inflammation exaggerates vascular dysfunction and induces further neuronal cell death (Dirnagl et al., 1999). Thus, post-ischemic inflammation is an essential process in the pathophysiology of ischemic stroke and is closely related to the prognosis after stroke (Dirnagl et al., 1999; Lo, 2010; Moskowitz et al., 2010; Iadecola and Anrather, 2011). In addition, inflammation is generally considered useful for the clearance of the large amount of debris caused by brain cell necrotic death (Moskowitz et al., 2010; Iadecola and Anrather, 2011). Inflammation, resolution of inflammation, and repair of neural damage are sequential pivotal events after stroke. To clarify the detailed mechanisms of each step of cerebral inflammation is indispensable to develop next generation therapies for ischemic stroke. The molecular basis of these steps is now being clarified by the recent accumulating evidences. We summarize these findings and discuss the principles of post-ischemic inflammation from beginning to end.

\section{INFLAMMATORY DAMPS}

Brain ischemia induces various large metabolic changes in brain cells. Hypoxic stress, nutrients stress, and ER stress will cause cell death and trigger post-ischemic inflammation. Although receptors for pathogens such as Toll-like receptors (TLRs) are thought to be involved in early step of inflammation, brain is a sterile organ. Thus, endogenous molecules, i.e., DAMPs derived from injured brain cells, must trigger the inflammatory response in immune cells (Table 1). These DAMPs induce the activation of TLRs and other pattern recognition receptors [receptor for advanced glycation end products (RAGE) and c-type lectin receptors], which promote inflammatory mediator expression and tissue injury (Figure 1; Tang et al., 2007; Yanai et al., 2009; Suzuki et al., 2013). Recent scientific advances have suggested the existence of various types of DAMPs in ischemic brain.

\section{NUCLEIC ACIDS AND NUCLEOTIDES}

Various intracellular components are released into the extracellular space by necrotic brain cell death. Among these, 
Table 1 | List of inflammatory DAMPs.

\begin{tabular}{|c|c|c|c|c|}
\hline & & DAMPs & Receptor & Reference \\
\hline \multirow[t]{9}{*}{ Signal 1} & Nucleic acid & Mitochondrial DNA & TLR9 & $\begin{array}{l}\text { Zhang etal. (2010), Sun etal. (2013), Maeda and Fadeel (2014), Walko et al. } \\
\text { (2014), Wenceslau etal. (2014) }\end{array}$ \\
\hline & & Self RNA, DNA & TLR7,9 & $\begin{array}{l}\text { Hyakkoku etal. (2010), Kawai and Akira (2010), Brea et al. (2011), Stevens et al } \\
\text { (2011), Leung etal. (2012) }\end{array}$ \\
\hline & Lipid & Carboxyalkylpyrroles & TLR2 & West etal. (2010) \\
\hline & & Oxidized phospholipids & CD36 & $\begin{array}{l}\text { Cho etal. (2005), Gao etal. (2006), Abe et al. (2010), Haider etal. (2011), Miller } \\
\text { et al. (2011), Ho etal. (2012), Matt etal. (2013) }\end{array}$ \\
\hline & Protein & HMGB1 & TLR2,4, RAGE & Qiu etal. (2008), Zhang etal. (2011) \\
\hline & & Peroxiredoxin & TLR2,4 & Shichita etal. (2012), Kuang etal. (2014) \\
\hline & & S100A8, A9 & TLR4 & Tsai et al. (2014) \\
\hline & & Mrp8, 14 & TLR4 & Loser etal. (2010) \\
\hline & & CIRP & TLR2,4 & Qiang et al. (2013) \\
\hline \multirow[t]{3}{*}{ Signal 2} & Nucleotide & ATP & $P 2 X, P 2 Y$ & $\begin{array}{l}\text { Martinon et al. (2002), Abulafia etal. (2009), Ceruti etal. (2009), Denes et al. } \\
\text { (2013), Fann etal. (2013), Yang etal. (2014), }\end{array}$ \\
\hline & Lipid & Phospholipids & $?$ & $\begin{array}{l}\text { Clemens etal. (1996), Bonventre et al. (1997), Muralikrishna Adibhatla and } \\
\text { Hatcher (2006), Shanta etal. (2012), Iyer etal. (2013), Zhong etal. (2013) }\end{array}$ \\
\hline & Protein & ASC specks & $?$ & Baroja-Mazo et al. (2014), Franklin et al. (2014) \\
\hline
\end{tabular}

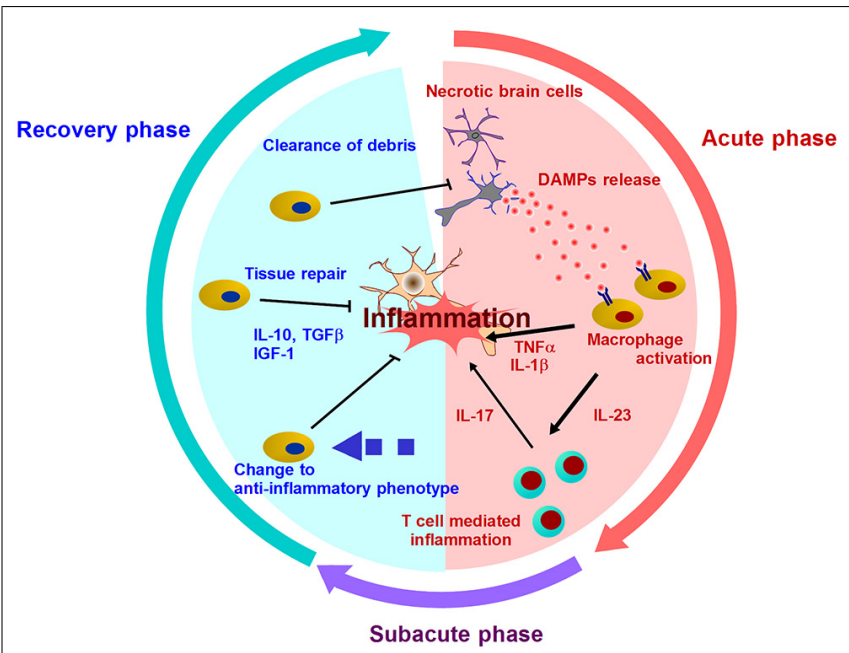

FIGURE 1 | Mechanisms of post-ischemic inflammation. DAMPS are released into extracellular compartment and activate infiltrating immune cells by two ways: Signal 1 (via the activation of pattern recognition receptor) and Signal 2 (via the activation of inflammasome). Various inflammatory cytokines promote neuronal injury, and induce further inflammation mediated by T cells in subacute phase. After days and week after stroke onset, the resolution of post-ischemic inflammation is brought by the clearance of debris including DAMPs or inflammatory mediators, and the production of anti-inflammatory molecules or neurotrophic factors. In this recover phase, inflammatory immune cells turn into neuroprotective cells.

nucleic acids and nucleotides are major DAMPs that have recently received much attention. Mitochondrial DNA released by cellular injury can be detected as DAMPs by immune cells, because mitochondria are considered to have a symbiotic origin that carries numerous characteristics resembling bacteria. Mitochondrial DNA is a sensor molecule of innate immunity by activating TLR9 and can be detected in cerebrospinal fluid after traumatic brain injury (Zhang et al., 2010; Walko et al., 2014). Vascular permeability is also increased by circulating mitochondrial DNA after injury (Sun et al., 2013; Wenceslau et al., 2014). Recently accumulated data indicates that mitochondrial DAMPs could be an important candidate for the trigger of post-ischemic inflammation, even if there is not yet any direct evidence (Maeda and Fadeel, 2014).

Self RNA and DNA (in complex with LL37 peptide) activate immune cells via TLR7 or TLR9 (Kawai and Akira, 2010). TLR7 is associated with the deterioration in ischemic stroke patients; in contrast, ischemic brain damage was not reduced in TLR9-deficient mice (Hyakkoku et al., 2010; Brea et al., 2011). Several reports demonstrate the implications of TLR7 and TLR9 in ischemic preconditioning. In these articles, the pretreatment using a TLR7 or TLR9 agonist reveals significant neuroprotection after cerebral ischemia by activating interferon regulatory factor 3/7- (IRF3/7)-induced type I interferon (IFN) signaling pathway (Stevens et al., 2011; Leung et al., 2012). Although the interaction between self nucleic acids and TLRs in the ischemic brain remains controversial, ischemic preconditioning via the TLR7 or TLR9 signaling pathway may represent a therapeutic strategy.

Purines (ATP and UTP) released from injured brain cells and their receptors, $\mathrm{P} 2 \mathrm{X}$ and $\mathrm{P} 2 \mathrm{Y}$, function as alerting signals in CNS (Ceruti et al., 2009). Importantly, ATP also activates inflammasomes, which are large multimolecular complexes that control the activity of the proteolytic enzyme caspase- 1 that cleaves pro-IL-1 $\beta$ to an active $17 \mathrm{kDa}$ form (Martinon et al., 2002). The activation of 
the NLRP1 or NALP3 inflammasome has been recently reported to promote post-ischemic inflammation and neuronal death (Abulafia et al., 2009; Fann et al., 2013; Yang et al., 2014). Because IL-1 $\beta$ produced from both infiltrating immune cells and brain cells is important (Denes et al., 2013), it should be clarified how the inflammasome is activated in ischemic brain or hematopoietic cells. Inhibition of the inflammasome activation pathway may be a possible therapeutic strategy for ischemic stroke.

\section{LIPIDS}

Various types of lipids are also important regulators of innate immunity. For example, oxidized low density lipoprotein (oxLDL) is a popular inflammatory mediator, which activates TLRs through binding with its receptor, CD36 (Stewart et al., 2010). Although the function of oxLDL in ischemic brain remains unclear, recent research has indicated that end products of lipid oxidation may be implicated in cerebral post-ischemic inflammation (Uchida, 2013). Carboxyalkylpyrroles, which are generated in inflammatory tissue, activate TLR2 and promote angiogenesis in ischemic organs (West et al., 2010). Oxidized phospholipids are also generated during cerebral inflammation and are considered to be DAMPs (Gao et al., 2006; Haider et al., 2011; Ho etal., 2012). Oxidized phospholipids are CD36 ligands that promote inflammation via TLR2 activation in ischemic brain (Cho etal., 2005; Abe et al., 2010). The recognition and endocytosis of oxidized lipids by pattern recognition receptors could regulate post-ischemic inflammation (Miller et al., 2011; Matt et al., 2013).

Phospholipids could also be inflammasome activators. Phospholipid metabolism is drastically altered by cerebral ischemia (Shanta et al., 2012). There are several reports showing the activation of phospholipase A2 (PLA2) in ischemic brain, which results in hydrolysis of membrane phospholipids (Clemens et al., 1996; Bonventre etal., 1997; Muralikrishna Adibhatla and Hatcher, 2006). Phospholipid hydrolysis and mitochondrial dysfunction induced by cerebral ischemia generate reactive oxygen species (ROS). Two recent studies have identified both ROS-dependent and ROS-independent pathways for inflammasome activation. The former is demonstrated by a charged phospholipid liposome that consecutively induces ROSdependent calcium influx and NLRP3 inflammasome activation (Zhong etal., 2013). In the latter case, mitochondrial cardiolipin has been reported to directly bind to and activate the NLRP3 inflammasome (Iyer et al., 2013). Thus, the metabolism and modification of lipids during cerebral ischemia may be closely associated with the post-ischemic inflammation start signal.

\section{PROTEINS}

High mobility group box 1 (HMGB1) and peroxiredoxin (Prx) family proteins are two major DAMPs in ischemic brain. There is a difference in the functional phase of these two proteins (Shichita etal., 2012). HMGB1, which is included in the nucleus of brain cells, is released extracellularly at the hyperacute phase (several hours after the stroke onset; Qiu et al., 2008). On the other hand, Prx family proteins function at the acute and subacute phases (12-72 $\mathrm{h}$ after the onset), especially in the penumbral area (Shichita etal., 2012). This is because Prx family protein expression is induced by an intracellular increase in ROS, which results from ischemic change. HMGB1 directly breaks down the blood-brain barrier and increases vascular permeability (Zhang et al., 2011). However, Prx directly induces the activation of infiltrating immune cells via TLR signaling. Ligustilide has been reported as a therapeutic candidate that suppresses cerebral post-ischemic inflammation by inhibiting the Prx/TLR4 signaling pathway (Kuang et al., 2014).

S100A8, S100A9, Mrp8, Mrp14, and cold-inducible RNA binding protein (CIRP) have also been reported to be protein DAMPs, although their relevance in post-ischemic inflammation has not yet been clarified (Loser et al., 2010; Qiang et al., 2013; Tsai et al., 2014). Inflammatory responses by these protein DAMPs occur through the activation of TLR2, TLR4, and RAGE. TLR2 and TLR4 signaling pathways are essential for sterile inflammation, including ischemic stroke (Chen et al., 2007). TLR2-blocking antibody is neuroprotective against ischemic brain injury (Ziegler et al., 2011). Similarly, resatorvid, which inhibits the TLR4 signaling pathway, attenuates ischemic brain injury and also suppresses Nox4-induced oxidative stress and neuronal apoptosis (Suzuki et al., 2012). It is also possible that DAMP-mediated TLR activation requires other adaptor molecules (Chun and Seong, 2010). CD14, a TLR4 co-receptor, may be implicated in post-ischemic inflammation (Reed-Geaghan et al., 2009). Heat shock protein gp96 is another candidate molecule that functions as an adaptor for both TLR2 and TLR4 (Yang et al., 2007).

It is not known whether protein DAMPs can activate inflammasomes. Recently, aggregated ASC (apoptosis-associated speck-like protein containing a caspase recruitment domain) has been reported to be released into the extracellular space after cell death and it activates inflammasomes in the surrounding immune cells (Baroja-Mazo et al., 2014; Franklin et al., 2014). Inflammasome activation, which occurs through ASC polymerization, results in caspase- 1 activation and pyroptotic cell death. Extracellularly released ASC is internalized by surrounding macrophages and induces lysosomal damage and inflammasome activation. These mechanisms of inflammasome activation remain to be elucidated in ischemic brain injury.

\section{OTHER INFLAMMATORY DAMPS}

Basic research may neglect the influence of aging and life habits by using healthy young rodents. These are important factors for the generation of DAMPs. Aging and continuous high serum glucose levels increase lipid peroxidation and AGEs in body systems (Basta et al., 2004; Cai et al., 2014). AGEs are proteins that are modified by sugar, through the Amadori and Maillard reaction. AGEs are found in chronic lesions; for example, the amyloid deposits that are surrounded by macrophages in patients with dialysis-related amyloidosis (Miyata et al., 1993). Thus, AGEs usually take a long time (more than a month) to generate; however, AGEs can be generated in a short period of time during inflammation (Weil, 2012). Glyoxal and glyceraldehyde induce AGE formation within 1 week (Takeuchi et al., 2001). In addition, the pivotal role of the RAGE in post-ischemic inflammation has been demonstrated (Muhammad et al., 2008). AGEs can be a potential DAMP, especially in aged human ischemic stroke patients. 


\section{INFLAMMATION SUPPRESSION AND RESOLUTION}

Activated immune cells and brain cells are the major players after various DAMPs trigger post-ischemic inflammation. These cells produce inflammatory cytokines, chemokines, and other cytotoxic mediators, and this leads to prolonged inflammation and progressive brain edema during several days after the stroke onset (Figure 1). However, post-ischemic inflammation rarely lasts for a long period of time, and the most intense inflammatory phase takes place within 7 days after stroke onset (Dirnagl et al., 1999; Iadecola and Anrather, 2011). In this phase, the number of infiltrating immune cells decreases remarkably, and remaining immune cells in ischemic brain produce anti-inflammatory or neurotrophic factors (Shichita etal., 2009; Smirkin etal., 2010). For example, the detailed mechanisms about the infiltration and the change to antiinflammatory phenotype of neutrophils have been recently clarified (Cuartero etal., 2013; Gorina etal., 2014). The period of cerebral post-ischemic inflammation always ends, and thus, the mechanisms of its resolution must exist in ischemic brain.

Three major points on the resolution of inflammation have been discussed in a recent publication (Buckley et al., 2012). These points are the production of anti-inflammatory mediators, the depletion of inflammatory mediators, and the induction of anti-inflammatory immune cells. After post-ischemic inflammation, infiltrating macrophages turn into anti-inflammatory macrophages, which produce neurotropic factors and clear necrotic debris. Inflammatory DAMPs will also be implicated in the induction of anti-inflammatory macrophages, although its mechanism still remains to be clarified. We introduce recent advantages of the relationship between post-ischemic inflammation and its resolution.

\section{ANTI-INFLAMMATORY MEDIATOR PRODUCTION}

Many molecules have been reported to be neuroprotective factors. However, most of these molecules failed to improve neurological deficits in ischemic stroke patients, even if they are effective in animal stroke models. It has been suggested that neuroprotection alone is not sufficient to improve the prognosis of human ischemic stroke. Anti-inflammatory mechanisms in the entire brain and how these mechanisms are triggered needs to be determined. Because most brain cells are dead in the ischemic region several days after the stroke onset, infiltrating immune cells and reactive glial cells could be major players in the tissue repair. In practice, accelerating their effect is a potential next generation therapeutic strategy, and direct in vivo reprogramming of reactive glial cells into functional neurons after cerebral injury by retroviral transduction of the NeuroD1 gene was recently reported (Guo et al., 2014).

IL-10 and TGF- $\beta$ are major anti-inflammatory molecules in various organ injuries. Both are produced by infiltrating immune cells and reactive glial cells after ischemic brain injury. Viral overexpression of IL-10 in ischemic brain is neuroprotective (Ooboshi et al., 2006). One recent report demonstrated the anti-inflammatory effect of TGF- $\beta$ by inhibiting excessive neuroinflammation during the subacute phase of brain ischemia (Cekanaviciute etal., 2014). Although the anti-inflammatory effects of IL-10 and TGF- $\beta$ have been pivotal, it remains to be clarified whether these effects last up to 1 week after the stroke onset (Pál et al., 2012). If the mechanisms for stimulating TGF- $\beta$ and IL-10 expression can be controlled, this may become a strong therapeutic method.

\section{DEPLETION OF INFLAMMATORY MEDIATORS AND CELLS}

Infiltrating immune cells and reactive glial cells produce various inflammatory mediators. TNF- $\alpha$ and IL- $1 \beta$ directly induce neuronal cell death. IL-23 and IL- $1 \beta$ activate T cell-mediated innate immunity and promote secondary ischemic damage during the subacute phase of ischemic brain injury (Shichita etal., 2009; Konoeda et al., 2010). The existence of these inflammatory mediators, including DAMPs, prolongs post-ischemic inflammation and will be a threat to neuronal survival and repair. However, inflammatory mediator degradation mechanisms remain mostly unknown. Inflammatory molecules may be degraded by some enzymes or consumed by receptor-mediated endocytosis. It is expected that nucleotides and lipids are rapidly metabolized in the ischemic brain, and transfer by the blood stream or cerebrospinal fluid (CSF) will help to scavenge inflammatory mediator. Further research should clarify the detailed mechanisms to scavenge inflammatory molecules produced in the ischemic brain, and antibody therapy will be a pivotal therapeutic method targeting this potential mechanism. TNF- $\alpha$ and IL-23 neutralizing antibody have been used clinically for rheumatoid arthritis and psoriasis patients, respectively. Natalizumab is the neutralizing antibody for integrin- $\alpha 4$, which is necessary for $\mathrm{T}$ cell infiltration into the inflammatory tissue, and has already been used for multiple sclerosis (Yednock et al., 1992). $\mathrm{T}$ cell depletion from ischemic brain has received attention as a potential next generation therapy for ischemic stroke (Meisel and Meisel, 2011; Wei et al., 2011). Thus, antibody therapies may be used to help treat ischemic stroke patients in the near future.

Activation of inflammasomes in immune cells induces the production of IL- $1 \beta$ in its mature form, and finally results in the rapid cell death of the same cells, which is called pyroptosis. Pyroptosis may be a possible mechanism for the clearance of inflammatory immune cells. This is supported by the fact that dying cells detected using the TdT-mediated dUTP nick end labeling (TUNEL) staining method include macrophages and glial cells in the ischemic brain (Mabuchi et al., 2000).

\section{INDUCTION OF IMMUNE CELL REPAIR}

The repair process for damaged brain tissues and regeneration of neural cells takes place during resolution of inflammation. It is difficult to separate this process from the anti-inflammatory mechanism, because they may overlap each other. We will further discuss neuroprotective factors and repairing the damage to immune cells.

\section{NEUROPROTECTIVE FACTORS}

Various growth factors are also produced by immune cells and glial cells (Gudi et al., 2011). Among these, IGF-1 and FGF-2 are produced by infiltrating macrophages and microglia during the recovery phase of ischemic brain injury (which occurs 
1 week after the stroke onset). IGF-1 and FGF-2 improve the neurological outcome by saving neuron and glial cells from cell death (Ness et al., 2004; Ikeda et al., 2005; Zhu et al., 2008; Hill etal., 2012; Lalancette-Hébert etal., 2012). IGF-1 also enhances repair after ischemic stroke by promoting neural regeneration, remyelination, and synaptogenesis (Lecker et al., 2007; Zhu etal., 2008, 2009; Kooijman etal., 2009). Further investigation should clarify the mechanisms of IGF-1 and FGF-2 induction in the ischemic brain. Recently, transfer of mesenchymal stem cells (MSCs) has been explored as a next generation therapy for ischemic stroke (Kalladka and Muir, 2014). MSCs produce various growth factors and promote neuronal survival and neurogenesis (Calió et al., 2014). By improving the transfer method, cell therapy may become a pivotal therapeutic strategy (Guo et al., 2013).

The neuroprotective effect of prostaglandin E2 (PGE2) and its receptor signaling pathway has received recent attention. PGE2 has an effect via four distinct $G$ protein-coupled EP receptors (E-prostanoid: EP1, EP2, EP3, and EP4). The activation of EP2 signaling has a neuroprotective effect in ischemic brain injury, which was shown in the significant increase in infarct volume in mice lacking the EP2 receptor (McCullough et al., 2004). Similarly, signaling via the EP4 receptor, which is expressed in both neurons and ischemic endothelial cells, has neuroprotective effects against ischemic brain injury (Liang et al., 2011). The administration of an EP4 agonist reduces infarct volume and neurological deficits. In a neonatal hypoxic-ischemic encephalopathy model, the inhibition of EP1 receptor signaling or the activation of EP2, EP3, and EP4 receptor signaling reveals attenuation of the ischemic injury (Taniguchi et al., 2011). PGE2 and EP receptor signaling pathways have various functions, which are dependent on distinct pathology of cerebral diseases (Furuyashiki and Narumiya, 2011). Targeting specific EP receptors in ischemic brain may become a novel therapeutic method.

Similar to PGE2, some lipids have been reported to have antiinflammatory effects and promote neuroregeneration (Serhan, 2014). Cerebral ischemia increases PLA2 activity, which results in the hydrolysis of phospholipids in the cellular membrane (Shanta et al., 2012). Although the PLA2 effect itself is cytotoxic because it disrupts the cellular membrane, PLA2 also generates docosahexaenoic acid (DHA) derivatives and lysophospholipids through phospholipid hydrolysis (Bonventre et al., 1997). Resolvin and Neuroprotectin have been investigated for their anti-inflammatory function in ischemic stroke (Marcheselli et al., 2003; Bazan, 2009). Lysophospholipids also increase in ischemic brain and promote neurite outgrowth (Ikeno et al., 2005; Spohr et al., 2011; Shanta et al., 2012). Regulating the effect of these lipids is expected for the resolution of post-ischemic inflammation.

\section{NEUROPROTECTIVE CELLS}

Inflammatory DAMPs activate glial cells and infiltrating immune cells to promote post-ischemic inflammation. Paradoxically, this mechanism results in the infiltrating macrophage cell death and also induces anti-inflammatory and tissue-repairing immune cells.

Immune cell activation also induces anti-inflammatory cells. These cells have been called M2 macrophages, in contrast to the inflammatory M1 macrophages. Many researches have described the M2 macrophage markers; these markers include: arginase1 (Arg1), chitinase313 (Ym), and Relm $\alpha$ (Fizz1). These markers are intracellular enzymes that are implicated in collagen synthesis and cell division; therefore, M2 enzymes are considered to promote tissue repair. Arg1 is the only marker that was reported to function as a neuroprotective enzyme (Estévez et al., 2006). However, these M2 markers may not be a good indicator for recovery after ischemic stroke. M2 markers are rapidly expressed in macrophages by TLR activation or other pattern recognition receptors, which also induce inflammatory cytokine expression (Hu et al., 2012). M2 markers appear in ischemic brain mostly during the same phase as the inflammatory mediators, the M1 markers, are expressed. In addition, the transfer of M2 marker positive-macrophages has not been reported to be sufficiently neuroprotective (Desestret et al., 2013).

During post-ischemic inflammation, some populations of macrophages and microglia become neuroprotective (LalancetteHébert etal., 2007). Galectin-1 has been suggested to be an inducer of anti-inflammatory macrophage/microglial cells (Starossom et al., 2012; Quintá et al., 2014). Galectin-1 is produced by astrocytes and has a neuroprotective effect against ischemic brain damage ( $\mathrm{Qu}$ etal., 2011). Thus, the resolution of post-ischemic inflammation can be enhanced by the induction of a specific macrophage/microglial cell population. However, it is not clear whether the M2 markers truly reflect the neuroprotective function of macrophages and microglial cells. Suppressing inflammation alone is not enough to protect the brain from ischemic injury. IGF-1 and FGF-2 production seems to be a good index of repairing function (Lalancette-Hébert et al., 2012).

Further study is required to clarify whether sufficient clearance of inflammatory mediators (including DAMPs) begins neuronal regeneration after ischemic stroke. A recent study has suggested that there is a relationship between TLR activation and neuronal repair (Bohacek et al., 2012). It is possible that DAMPs triggers the secondary signals, which lead to resolution of post-ischemic inflammation, even if the primary signals via pattern recognition receptors promote ischemic damage. What is this mechanism? The role of immune cells, other than macrophages and microglia in part of the repair process, is not fully understood. This understanding may be critical for the establishment of next generation therapies for ischemic stroke.

\section{CONCLUSION}

Immunity and various physiological mechanisms are implicated in the triggering, persistence, and resolution of post-ischemic inflammation. Recent accumulating evidences clarify the complexity of these mechanisms to understand the entire mechanisms. They will show promising potential targets to develop therapies for ischemic stroke.

\section{REFERENCES}

Abe, T., Shimamura, M., Jackman, K., Kurinami, H., Anrather, J., Zhou, P., et al. (2010). Key role of CD36 in Toll-like receptor 2 signaling in cerebral ischemia. Stroke 41, 898-904. doi: 10.1161/STROKEAHA.109.572552 
Abulafia, D. P., de Rivero Vaccari, J. P., Lozano, J. D., Lotocki, G., Keane, R. W., and Dietrich, W. D. (2009). Inhibition of the inflammasome complex reduces the inflammatory response after thromboembolic stroke in mice. J. Cereb. Blood Flow Metab. 29, 534-544. doi: 10.1038/jcbfm.2008.143

Baroja-Mazo, A., Martín-Sánchez, F., Gomez, A., Martínez, C. M., Amores-Iniesta J., Compan, V., et al. (2014). The NLRP3 inflammasome is released as a particulate danger signal that amplifies the inflammatory response. Nat. Immunol. 15, 738748. doi: 10.1038/ni.2919

Basta, G., Schmidt, A. M., and De Caterina, R. (2004). Advanced glycation end products and vascular inflammation: implications for accelerated atherosclerosis in diabetes. Cardiovasc. Res. 63, 582-592. doi: 10.1016/j.cardiores.2004.05.001

Bazan, N. G. (2009). Neuroprotectin D1-mediated anti-inflammatory and survival signaling in stroke, retinal degenerations, and Alzheimer's disease. J. Lipid Res. 50, S400-S405. doi: 10.1194/jlr.R800068-JLR200

Bohacek, I., Cordeau, P., Lalancette-Hébert, M., Gorup, D., Weng, Y. C., Gajovic, S., et al. (2012). Toll-like receptor 2 deficiency leads to delayed exacerbation of ischemic injury. J. Neuroinflammation 9, 191. doi: 10.1186/1742 2094-9-191

Bonventre, J. V., Huang, Z., Taheri, M. R., O’Leary, E., Li, E., Moskowitz, M. A. et al. (1997). Reduced fertility and postischaemic brain injury in mice deficient in cytosolic phospholipase A2. Nature 390, 622-625. doi: 10.1038/37635

Brea, D., Sobrino, T., Rodríguez-Yáñez, M., Ramos-Cabrer, P., Agulla, J., RodríguezGonzález, R., et al. (2011). Toll-like receptors 7 and 8 expression is associated with poor outcome and greater inflammatory response in acute ischemic stroke. Clin. Imunol. 139, 193-198. doi: 10.1016/j.clim.2011.02.001

Buckley, C. D., Gilroy, D. W., Serhan, C. N., Stockinger, B., and Tak, P. P. (2012). The resolution of inflammation. Nat. Rev. Immunol. 13, 59-66. doi: 10.1038/nri3362

Cai, W., Uribarri, J., Zhu, L., Chen, X., Swamy, S., Zhao, Z., et al. (2014). Oral glycotoxins are a modifiable cause of dementia and the metabolic syndrome in mice and humans. Proc. Natl. Acad. Sci. U.S.A. 111, 4940-4945. doi: 10.1073/pnas.1316013111

Calió, M. L., Marinho, D. S., Ko, G. M., Ribeiro, R. R., Carbonel, A. F., Oyama, L. M., et al. (2014). Transplantation of bone marrow mesenchymal stem cells decreases oxidative stress, apoptosis, and hippocampal damage in brain of a spontaneous stroke model. Free Radic. Biol. Med. 70, 141-154. doi 10.1016/j.freeradbiomed.2014.01.024

Cekanaviciute, E., Fathali, N., Doyle, K. P., Williams, A. M., Han, J., and Buckwalter, M. S. (2014). Astrocytic transforming growth factor-beta signaling reduces subacute neuroinflammation after stroke in mice. Glia 62, 1227-1240. doi: 10.1002/glia.22675

Ceruti, S., Villa, G., Genovese, T., Mazzon, E., Longhi, R., Rosa, P., et al. (2009). The P2Y-like receptor GPR17 as a sensor of damage and a new potential target in spinal cord injury. Brain 132(Pt 8), 2206-2218. doi: 10.1093/brain/ awp 147

Chen, C. J., Kono, H., Golenbock, D., Reed, G., Akira, S., and Rock, K. L. (2007) Identification of a key pathway required for the sterile inflammatory response triggered by dying cells. Nat. Med. 13, 851-856. doi: 10.1038/nm1603

Cho, S., Park, E. M., Febbraio, M., Anrather, J., Park, L., Racchumi, G., et al. (2005). The class B scavenger receptor CD36 mediates free radical production and tissue injury in cerebral ischemia. J. Neurosci. 25, 2504-2512. doi: 10.1523/JNEUROSCI.0035-05.2005

Chun, K. H., and Seong, S. Y. (2010). CD14 but not MD2 transmit signals from DAMP. Int. Immunopharmaol. 10, 98-106. doi: 10.1016/j.intimp.2009.10.002

Clemens, J. A., Stephenson, D. T., Smalstig, E. B., Roberts, E. F., Johnstone, E. M., Sharp, J. D., et al. (1996). Reactive glia express cytosolic phospholipase A2 after transient global forebrain ischemia in the rat. Stroke 27, 527-535. doi: 10.1161/01.STR.27.3.527

Cuartero, M. I., Ballesteros, I., Moraga, A., Nombela, F., Vivancos, J., Hamilton, J. A., et al. (2013). N2 neutrophils, novel players in brain inflammation after stroke: modulation by the PPARg agonist rosiglitazone. Stroke 44, 3498-3508. doi: 10.1161/STROKEAHA.113.002470

Denes, A., Wilkinson, F., Bigger, B., Chu, M., Rothwell, N. J., and Allan, S. M. (2013). Central and haematopoietic interleukin-1 both contribute to ischaemic brain injury. Dis. Model. Mech. 6, 1043-1048. doi: 10.1242/dmm.011601

Desestret, V., Riou, A., Chauveau, F., Cho, T. H., Devillard, E., Marinescu, M., et al. (2013). In vitro and in vivo models of cerebral ischemia show discrepancy in therapeutics effects of M2 macrophages. PLoS ONE. 8:e67063. doi: 10.1371/journal.pone.0067063
Dirnagl, U., Iadecola, C., and Moskowitz, M. A. (1999). Pathobiology of ischaemic stroke: an integrated view. Trends Neurosci. 22, 391-397. doi: 10.1016/S01662236(99)01401-0

Estévez, A. G., Sahawneh, M. A., Lange, P. S., Bae, N., Egea, M., and Ratan, R. R. (2006). Arginase 1 regulation of nitric oxide production is key to survival of trophic factor-deprived motor neurons. J. Neurosci. 26, 8512-8516. doi: 10.1523/JNEUROSCI.0728-06.2006

Fann, D. Y., Lee, S. Y., Manzanero, S., Tang, S. C., Gelderblom, M., Chunduri, P., et al. (2013). Intravenous immunoglobulin suppresses NLRP1 and NLRP3 inflammasome-mediated neuronal death in ischemic stroke. Cell Death Dis. 4, e790. doi: 10.1038/cddis.2013.326

Franklin, B. S., Bossaller, L., De Nardo, D., Ratter, J. M., Stutz, A., Engels, G., etal. (2014). The adaptor ASC has extracellular and 'prionoid' activities that propagate inflammation. Nat. Immunol. 15, 727-737. doi: 10.1038/ ni.2913

Furuyashiki, T., and Narumiya, S. (2011). Stress responses: the contribution of prostaglandin $\mathrm{E}_{2}$ and its receptors. Nat. Rev. Endocrinol. 7, 163-175. doi: 10.1038/nrendo.2010.194

Gao, S., Zhang, R., Greenberg, M. E., Sun, M., Chen, X., Levison, B. S., et al. (2006). Phospholipid hydroxyalkenals, a subset of recently discovered endogenous CD36 ligands, spontaneously generate novel furan-containing phospholipids lacking CD36 binding activity in vivo. J. Biol. Chem. 281, 31298-31308. doi: 10.1074/jbc.M604039200

Gorina, R., Lyck, R., Vestweber, D., and Engelhardt, B. (2014). $\beta 2$ integrin-mediated crawling on endothelial ICAM-1 and ICAM-2 is a prerequisite for transcellular neutrophil diapedesis across the inflamed blood-brain barrier. J. Immunol. 192, 324-337. doi: 10.4049/jimmunol.1300858

Gudi, V., Škuljec, J., Yildiz, Ö., Frichert, K., Skripuletz, T., Moharregh-Khiabani, D., et al. (2011). Spatial and temporal profiles of growth factor expression during CNS demyelination reveal the dynamics of repair priming. PLoS ONE 6:e22623. doi: 10.1371/journal.pone.0022623

Guo, L., Ge, J., Wang, S., Zhou, Y., Wang, X., and Wu, Y. (2013). A novel method for efficient delivery of stem cells to the ischemic brain. Stem Cell Res. Ther. 4, 116. doi: $10.1186 /$ scrt327

Guo, Z., Zhang, L., Wu, Z., Chen, Y., Wang, F., and Chen, G. (2014). In Vivo direct reprogramming of reactive glial cells into functional neurons after brain injury and in an Alzheimer's disease model. Cell Stem Cell 14, 188-202. doi: 10.1016/j.stem.2013.12.001

Haider, L., Fischer, M. T., Frishcer, J. M., Bauer, J., Höftberger, R., Botond, G., et al. (2011). Oxidative damage in multiple sclerosis lesions. Brain 134(Pt 7), 1914-1924. doi: 10.1093/brain/awr128

Hill, J. J., Jin, K., Mao, X. O., Xie, L., and Greenberg, D. A. (2012). Intracerebral chondroitinase $\mathrm{ABC}$ and heparan sulfate proteoglycan glypican improve outcome from chronic stroke in rats. Proc. Natl. Acad. Sci. U.S.A. 109, 9155-9160. doi: 10.1073/pnas.1205697109

Ho, P. P., Kanter, J. L., Johnson, A. M., Srinagesh, H. K., Chang, E. J., Purdy, T. M., et al. (2012). Identification of naturally occurring fatty acids of the myelin sheath that resolve neuroinflammation. Sci. Transl. Med. 4, 137ra73. doi: 10.1126/scitranslmed.3003831

Hu, X., Li, P., Guo, Y., Wang, H., Leak, R. K., Chen, S., et al. (2012). Microglia/macrophage polarization dynamics reveal novel mechanism of injury expansion after focal cerebral ischemia. Stroke 43, 3063-3070. doi: 10.1161/STROKEAHA.112.659656

Hyakkoku, K., Hamanaka, J., Tsuruma, K., Shimazawa, M., Tanaka, H., Uematsu, S., et al. (2010). Toll-like receptor 4 (TLR4), but not TLR3 or TLR9, knock-out mice have neuroprotective effects against focal cerebral ischemia. Neuroscience 171, 258-267. doi: 10.1016/j.neuroscience.2010.08.054

Iadecola, C., and Anrather, J. (2011). The immunology of stroke: from mechanisms to translation. Nat. Med. 17, 796-808. doi: 10.1038/nm.2399

Ikeda, N., Nonoguchi, N., Zhao, M. Z., Watanabe, T., Kajimoto, Y., Furutama, D., et al. (2005). Bone marrow stromal cells that enhanced fibroblast growth factor-2 secretion by herpes simplex virus vector improve neurological outcome after transient focal cerebral ischemia in rats. Stroke 36, 2725-2730. doi: 10.1161/01.STR.0000190006.88896.d3

Ikeno, Y., Konno, N., Cheon, S. H., Bolchi, A., Ottonello, S., Kitamoto, K., et al. (2005). Secretory phospholipases A2 induce neurite outgrowth in PC12 cells through lysophosphatidylcholine generation and activation of G2A receptor. $J$. Biol. Chem. 280, 28044-28052. doi: 10.1074/jbc.M503343200 
Iyer, S. S., He, Q., Janczy, J. R., Elliott, E. I., Zhong, Z., Olivier, A. K., et al. (2013). Mitochondrial cardiolipin is required for Nlrp3 inflammasome activation. Immunity 39, 311-323. doi: 10.1016/j.immuni.2013.08.001

Kalladka, D., and Muir, K. W. (2014). Brain repair: cell therapy in stroke. Stem Cells Cloning 7, 31-44.

Kawai, T., and Akira, S. (2010). The role of pattern-recognition receptors in innate immunity: update on Toll-like receptors. Nat. Immunol. 11, 373-384. doi: 10.1038/ni.1863

Konoeda, F., Shichita, T., Yoshida, H., Sugiyama, Y., Muto, G., Hasegawa, E., et al. (2010). Therapeutic effect of IL-12/23 and their signaling pathway blockade on brain ischemia model. Biochem. Biophys. Res. Commun. 402, 500-506. doi: 10.1016/j.bbrc.2010.10.058

Kooijman, R., Sarre, S., Michotte, Y., and De Keyser, J. (2009). Insulin-like growth factor I: a potential neuroprotective compound for the treatment of acute ischemic stroke? Stroke 40, e83-e88. doi: 10.1161/STROKEAHA.108.528356

Kuang, X., Wang, L. F., Yu, L., Li, Y. J., Wang, Y. N., He, Q., etal. (2014). Ligustilide ameliorates neuroinflammation and brain injury in focal cerebral ischemia/reperfusion rats: involvement of inhibition of TLR4/peroxiredoxin 6 signaling. Free Radic. Biol. Med. 71, 165-175. doi 10.1016/j.freeradbiomed.2014.03.028

Lalancette-Hébert, M., Gowing, G., Simard, A., Weng, Y. C., and Kriz, J. (2007). Selective ablation of proliferating microglial cells exacerbates ischemic injury in the brain. J. Neurosci. 27, 2596-2605. doi: 10.1523/JNEUROSCI.5360 $-06.2007$

Lalancette-Hébert, M., Swarup, V., Beaulieu, J. M., Bohacek, I., Abdelhamid, E., Weng, Y. C., et al. (2012). Galectin-3 is required for resident microglia activation and proliferation in response to ischemic injury. J. Neurosci. 32, 10383-10395. doi: 10.1523/JNEUROSCI.1498-12.2012

Lecker, R. R., Soldner, F., Velasco, I., Gavin, D. K., Androutsellis-Theotokis, A., and McKay, R. D. (2007). Long-lasting regeneration after ischemia in the cerebral cortex. Stroke 38, 153-161. doi: 10.1161/01.STR.0000252156.65953.a9

Leung, P. Y., Stevens, S. L., Packard, A. E., Lessov, N. S., Yang, T., Conrad, V. K., et al. (2012). Toll-like receptor 7 preconditioning induces robust neuroprotection against stroke by a novel type I interferon-mediated mechanism. Stroke 43, 13831389. doi: 10.1161/STROKEAHA.111.641522

Liang, X., Lin, L., Woodling, N. S., Wang, Q., Anacker, C., Pan, T., et al. (2011). Signaling via the prostaglandin E2 receptor EP4 exerts neuronal and vascular protection in a mouse model of cerebral ischemia. J. Clin. Invest. 121, 4362-4371. doi: $10.1172 /$ JCI46279

Lo, E. H. (2010). Degeneration and repair in central nervous system disease. Nat. Med. 16, 1205-1209. doi: 10.1038/nm.2226

Loser, K., Vogl, T., Voskort, M., Leuken, A., Kupas, V., Nacken, W., et al. (2010). The Toll-like receptor 4 ligands Mrp8 and Mrp14 are crucial in the development of autoreactive $\mathrm{CD}^{+}{ }^{+}$T cells. Nat. Med. 16, 713-717. doi: 10.1038/nm. 2150

Mabuchi, T., Kitagawa, K., Ohtsuki, T., Kuwabara, K., Yagita, Y., Yanagihara, T., et al. (2000). Contribution of microglia/macrophages to expansion of infarction and response of oligodendrocytes after focal cerebral ischemia in rats. Stroke 31 1735-1743. doi: 10.1161/01.STR.31.7.1735

Maeda, A., and Fadeel, B. (2014). Mitochondria released by cells undergoing TNF- $\alpha$-induced necroptosis act as danger signals. Cell Death Dis. 5, e1312. doi: $10.1038 /$ cddis. 2014.277

Marcheselli, V. L., Hong, S., Lukiw, W. J., Tian, X. H., Gronert, K., Musto, A., et al. (2003). Novel docosanoids inhibit brain ischemia-reperfusion-mediated leukocyte infiltration and pro-inflammatory gene expression. J. Biol. Chem. 278, 43807-43817. doi: 10.1074/jbc.M305841200

Martinon, F., Burns, K., and Tschopp, J. (2002). The inflammasome: a molecular platform triggering activation of inflammatory caspases and processing of proILB. Mol. Cell 10, 417-426. doi: 10.1016/S1097-2765(02)00599-3

Matt, U., Sharif, O., Martins, R., Furtner, T., Langeberg, L., Gawish, R., et al. (2013). WAVE1 mediates suppression of phagocytosis by phospholipid-derived DAMPs. J. Clin. Invest. 123, 3014-3024. doi: 10.1172/JCI60681

McCullough, L., Wu, L., Haughey, N., Liang, X., Hand, T., Wang, Q., et al. (2004). Neuroprotective function of the $\mathrm{PGE}_{2} \mathrm{EP} 2$ receptor in cerebral ischemia. $J$ Neurosci. 24, 257-268. doi: 10.1523/JNEUROSCI.4485-03.2004

Meisel, C., and Meisel, A. (2011). Suppressing immunosuppression after stroke. N. Engl. J. Med. 365, 2134-2136. doi: 10.1056/NEJMcibr11 12454
Miller, Y. I., Choi, S. H., Wiesner, P., Fang, L., Harkewicz, R., Hartvigsen, K., et al. (2011). Oxidation-specific epitopes are danger-associated molecular patterns recognized by pattern recognition receptors of innate immunity. Circ. Res. 108, 235-248. doi: 10.1161/CIRCRESAHA.110.223875

Miyata, T., Oda, O., Inagi, R., Iida, Y., Araki, N., Yamada, N., et al. (1993). beta 2-Microglobulin modified with advanced glycation end products is a major component of hemodialysis-associated amyloidosis. J. Clin. Invest. 92, 1243-1252. doi: 10.1172/JCI116696

Moskowitz, M. A., Lo, E. H., and Iadecola, C. (2010). The science of stroke: mechanisms in search of treatments. Neuron 67, 181-198. doi: 10.1016/j.neuron.2010.07.002

Muhammad, S., Barakat, W., Stoyanov, S., Murikinati, S., Yang, H., Tracey, K. J., et al. (2008). The HMGB1 receptor RAGE mediates ischemic brain damage. $J$. Neurosci. 28, 12023-12031. doi: 10.1523/JNEUROSCI.2435-08.2008

Muralikrishna Adibhatla, R., and Hatcher, J. F. (2006). Phospholipase A2, reactive oxygen species, and lipid peroxidation in cerebral ischemia. Free Radic. Biol. Med. 40, 376-387. doi: 10.1016/j.freeradbiomed.2005.08.044

Ness, J. K., Scaduto, R. C. Jr., and Wood, T. L. (2004). IGF-I prevents glutamatemediated bax translocation and cytochrome $\mathrm{C}$ release in $\mathrm{O}^{+}$oligodendrocyte progenitors. Glia 46, 183-194. doi: 10.1002/glia.10360

Ooboshi, H., Ibayashi, S., Shichita, T., Kumai, Y., Takada, J., Ago, T., et al. (2006). Postischemic gene transfer of interleukin-10 protects against both focal and global brain ischemia. Circulation 111, 913-919. doi: 10.1161/01.CIR.0000155622.68580.DC

Pál, G., Vincze, C., Renner, É., Wappler, E. A., Nagy, Z., Lovas, G., et al. (2012). Time course, distribution and cell types of induction of transforming growth factor betas following middle cerebral artery occlusion in the rat brain. PLoS ONE 7:e46731. doi: 10.1371/journal.pone.0046731

Qiang, X., Yang, W. L., Wu, R., Zhou, M., Jacob, A., Dong, W., et al. (2013). Cold-inducible RNA-binding protein (CIRP) triggers inflammatory responses in hemorrhagic shock and sepsis. Nat. Med. 19, 1489-1495. doi: 10.1038/nm.3368

Qiu, J., Nishimura, M., Wang, Y., Sims, J. R., Qiu, S., Savitz, S. I., et al. (2008). Early release of HMGB-1 from neurons after the onset of brain ischemia. J. Cereb. Blood Flow Metab. 28, 927-938. doi: 10.1038/s.j.jcbfm. 9600582

Qu, W. S., Wang, Y. H., Ma, J. F., Tian, D. S., Zhang, Q., Pan, D. J., etal. (2011). Galectin-1 attenuates astrogliosis-associated injuries and improves recovery of rats following focal cerebral ischemia. J. Neurochem. 116, 217-226. doi: 10.1111/j.1471-4159.2010.07095.x

Quintá, H. R., Pasquini, J. M., Rabinovich, G. A., and Pasquini, L. A. (2014). Glycandependent binding of galectin-1 to neuropilin-1 promotes axonal regeneration after spinal cord injury. Cell Death. Differ. 21, 941-955. doi: 10.1038/cdd.2014.14

Reed-Geaghan, E. G., Savage, J. C., Hise, A. G., and Landreth, G. E. (2009). CD14 and toll-like receptors 2 and 4 are required for fibrillar A $\beta$-stimulated microglial activation. J. Neurosci. 29, 11982-11992. doi: 10.1523/JNEUROSCI.3158-09.2009 Serhan, C. N. (2014). Pro-resolving lipid mediators are leads for resolution physiology. Nature 510, 92-101. doi: 10.1038/nature13479

Shanta, S. R., Choi, C. S., Lee, J. H., Shin, C. Y., Kim, Y. J., Kim, K. H., et al. (2012). Global changes in phospholipids identified by MALDI MS in rats with focal cerebral ischemia. J. Lipid Res. 53, 1823-1831. doi: 10.1194/jlr. M022558

Shichita, T., Hasegawa, E., Kimura, A., Morita, R., Sakaguchi, R., Takada, I., et al. (2012). Peroxiredoxin family proteins are key initiators of post-ischemic inflammation in the brain. Nat. Med. 18, 911-917. doi: 10.1038/nm.2749

Shichita, T., Sugiyama, Y., Ooboshi, H., Sugimori, H., Nakagawa, R., Takada, I., et al. (2009). Pivotal role of cerebral interleukin-17-producing $\gamma \delta$ T cells in the delayed phase of ischemic brain injury. Nat. Med. 215, 946-950. doi: 10.1038/nm.1999

Smirkin, A., Matsumoto, H., Takahashi, H., Inoue, A., Tagawa, M., Ohue, S., et al. (2010). Iba $1^{+} / \mathrm{NG}^{+}$macrophage-like cells expressing a variety of neuroprotective factors ameliorate ischemic damage of the brain. J. Cereb. Blood Flow Metab. 30, 603-615. doi: 10.1038/jcbfm.2009.233

Spohr, T. C., Dezonne, R. S., Rehen, S. K., and Gomes, F. C. (2011). Astrocytes treated by lysophosphatidic acid induce axonal outgrowth of cortical progenitors through extracellular matrix protein and epidermal growth factor signaling pathway. $J$. Neurochem. 119, 113-123. doi: 10.1111/j.1471-4159.2011.07421.x

Starossom, S. C., Mascanfroni, I. D., Imitola, J., Cao, L., Raddassi, K., Hernandez, S. F., et al. (2012). Galectin-1 deactivates classically activated microglia and protects from inflammation-induced neurodegeneration. Immunity 37, 249-263. doi: 10.1016/j.immuni.2012.05.023 
Stevens, S. L., Leung, P. Y., Vartanian, K. B., Gopalan, B., Yang, T., Simon, R. P., et al. (2011). Multiple preconditioning paradigms converge on interferon regulatory factor-dependent signaling to promote tolerance to ischemic brain injury. J. Neurosci. 31, 8456-8463. doi: 10.1523/JNEUROSCI.082111.2011

Stewart, C. R., Stuart, L. M., Wilkinson, K., van Gils, J. M., Deng, J., Halle, A., et al. (2010). CD36 ligands promote sterile inflammation through assembly of a Toll-like receptor 4 and 6 heterodimer. Nat. Immunol. 11, 155-161. doi: 10.1038/ni.1836

Sun, S., Sursal, T., Adibnia, Y., Zhao, C., Zheng, Y., Li, H., et al. (2013). Mitochondrial DAMPs increase endothelial permeability through neutrophil dependent and independent pathways. PLOS ONE 8:e59989. doi: 10.1371/journal.pone. 0059989

Suzuki, Y., Hattori, K., Hamanaka, J., Murase, T., Egashira, Y., Mishiro, K., et al. (2012). Pharmacological inhibition of TLR4-NOX4 signal protects against neuronal death in transient focal ischemia. Sci. Rep. 2, 896. doi: 10.1038/srep00896

Suzuki, Y., Nakano, Y., Mishiro, K., Takagi, T., Tsuruma, K., Nakamura, M., et al. (2013). Involvement of Mincle and Syk in the changes to innate immunity after ischemic stroke. Sci. Rep. 3, 3177. doi: 10.1038/srep03177

Takeuchi, M., Yanase, Y., Matsuura, N., Yamagishi Si, S., Kameda, Y., Bucala, R., et al. (2001). Immunological detection of a novel advanced glycation end-product. Mol. Med. 7, 783-791.

Tang, S. C., Arumugam, T. V., Xu, X., Cheng, A., Mughal, M. R., Jo, D. G., et al. (2007). Pivotal role for neuronal Toll-like receptors in ischemic brain injury and functional deficits. Proc. Natl. Acad. Sci. U.S.A. 104, 13798-13803. doi: 10.1073/pnas.0702553104

Taniguchi, H., Anacker, C., Suarez-Mier, G. B., Wang, Q., and Andreasson, K. (2011). Function of prostaglandin E2 EP receptors in the acute outcome of rodent hypoxic ischemic encephalopathy. Neurosci. Lett. 504, 185-190. doi: 10.1016/j.neulet.2011.09.005

Tsai, S. Y., Segovia, J. A., Chang, T. H., Morris, I. R., Berton, M. T., Tessier, P. A., et al. (2014). DAMP molecule S100A9 acts as a molecular pattern to enhance inflammation during influenza A virus infection: role of DDX21-TRIF-TLR4MyD88 pathway. PLoS Pathog. 10:e1003848. doi: 10.1371/journal.ppat.1003848

Uchida, K. (2013). Redox-derived damage-associated molecular patterns: ligand function of lipid peroxidation adducts. Redox Biol. 1, 94-96. doi: 10.1016/j.redox.2012.12.005

Walko, T. D. 3rd, Bola, R. A., Hong, J. D., Au, A. K., Bell, M. J., Kochanek, P. M., et al. (2014). Cerebrospinal fluid mitochondrial DNA: a novel DAMP in pediatric traumatic brain injury. Shock 41, 499-503. doi: 10.1097/SHK.00000000 00000160

Wei, Y., Yemisci, M., Kim, H. H., Yung, L. M., Shin, H. K., Hwang, S. K., et al. (2011). Fingolimod provides long-term protection in rodent models of cerebral ischemia. Ann. Neurol. 69, 119-129. doi: 10.1002/ana.22186

Weil, Z. M. (2012). Ischemia-induced hyperglycemia: consequences, neuroendocrine regulation, and a role for RAGE. Horm. Behav. 62, 280-285. doi: 10.1016/j.yhbeh.2012.04.001

Wenceslau, C. F., McCarthy, C. G., Szasz, T., Spitler, K., Goulopoulou, S., Webb, R. C., et al. (2014). Mitochondrial damage-associated molecular patterns and vascular function. Eur. Heart J. 35, 1172-1177. doi: 10.1093/eurheartj/ehu047

West, X. Z., Malinin, N. L., Merkulova, A. A., Tischenko, M., Kerr, B. A., Borden, E. C., et al. (2010). Oxidative stress induces angiogenesis by activating TLR2 with novel endogenous ligands. Nature 467, 972-976. doi: 10.1038/nature09421
Yanai, H., Ban, T., Wang, Z., Choi, M. K., Kawamura, T., Negishi, H., et al. (2009). HMGB proteins function as universal sentinels for nucleicacid-mediated innate immune responses. Nature 462, 99-103. doi: 10.1038/ nature 08512

Yang, F., Wang, Z., Wei, X., Han, H., Meng, X., Zhang, Y., et al. (2014). NLRP3 deficiency ameliorates neurovascular damage in experimental ischemic stroke. J. Cereb. Blood Flow Metab. 34, 660-667. doi: 10.1038/jcbfm.2013.242

Yang, Y., Liu, B., Dai, J., Srivastava, P. K., Zammit, D. J., Lefrançois, L., et al. (2007). Heat shock protein gp96 is a master chaperone for Toll-like receptors and is important in the innate function of macrophages. Immunity 26, 215-226. doi: 10.1016/j.immuni.2006.12.005

Yednock, T. A., Cannon, C., Fritz, L. C., Sanchez-Madrid, F., Steinman, L., and Karin, N. (1992). Prevention of experimental autoimmune encephalomyelitis by antibodies against $\alpha 4 \beta 1$ integrin. Nature 356, 63-66. doi: 10.1038/ $356063 \mathrm{a} 0$

Zhang, J., Takahashi, H. K., Liu, K., Wake, H., Liu, R., Maruo, T., et al. (2011). Anti-high mobility group box-1 monoclonal antibody protects the blood-brain barrier from ischemia-induced disruption in rats. Stroke 42, 1420-1428. doi: 10.1161/STROKEAHA.110.598334

Zhang, Q., Raoof, M., Chen, Y., Sumi, Y., Sursal, T., Junger, W., et al. (2010). Circulating mitochondrial DAMPs cause inflammatory responses to injury. Nature 464, 104-107. doi: 10.1038/nature08780

Zhong, Z., Zhai, Y., Liang, S., Mori, Y., Han, R., Sutterwala, F. S., et al. (2013). TRPM2 links oxidative stress to NLRP3 inflammasome activation. Nat. Commun. 4, 1611. doi: $10.1038 /$ ncomms 2608

Zhu, W., Fan, Y., Frenzel, T., Gasmi, M., Bartus, R. T., Young, W. L., et al. (2008). Insulin growth factor-1 gene transfer enhances neurovascular remodeling and improves long-term stroke outcome in mice. Stroke 39, 1254-1261. doi: 10.1161/STROKEAHA.107.500801

Zhu, W., Fan, Y., Hao, Q., Shen, F., Hashimoto, T., Yang, G. Y., et al. (2009). Postischemic IGF-1 gene transfer promotes neurovascular regeneration after experimental stroke. J. Cereb. Blood Flow Metab. 29, 1528-1537. doi: $10.1038 /$ jcbfm. 2009.75

Ziegler, G., Freyer, D., Harhausen, D., Khojasteh, U., Nietfeld, W., and Trendelenburg, G. (2011). Blocking TLR2 in vivo protects against accumulation of inflammatory cells and neuronal injury in experimental stroke. J. Cereb. Blood Flow Metab. 31, 757-766. doi: 10.1038/jcbfm.2010.161

Conflict of Interest Statement: The authors declare that the research was conducted in the absence of any commercial or financial relationships that could be construed as a potential conflict of interest.

Received: 28 July 2014; accepted: 23 September 2014; published online: 14 October 2014.

Citation: Shichita T, Ito $M$ and Yoshimura A (2014) Post-ischemic inflammation regulates neural damage and protection. Front. Cell. Neurosci. 8:319. doi: 10.3389/fncel.2014.00319

This article was submitted to the journal Frontiers in Cellular Neuroscience.

Copyright (C) 2014 Shichita, Ito and Yoshimura. This is an open-access article distributed under the terms of the Creative Commons Attribution License (CC BY). The use, distribution or reproduction in other forums is permitted, provided the original author(s) or licensor are credited and that the original publication in this journal is cited, in accordance with accepted academic practice. No use, distribution or reproduction is permitted which does not comply with these terms. 